\title{
Effect of glutamine supplement on chemo-radiation induced mucositis in head and neck cancer patients: a prospective study
}

\author{
Bishnu Prasad Das, Jitendra Patra* \\ Department of Radiotherapy, Maharaja Krishna Chandra Gajapati Medical College. Berhampur, Odisha, India \\ Received: 27 January 2020 \\ Revised: 11 February 2020 \\ Accepted: 12 February 2020 \\ *Correspondence: \\ Dr. Jitendra Patra, \\ Email: dr.jiten83@gmail.com \\ Copyright: (C) the author(s), publisher and licensee Medip Academy. This is an open-access article distributed under \\ the terms of the Creative Commons Attribution Non-Commercial License, which permits unrestricted non-commercial \\ use, distribution, and reproduction in any medium, provided the original work is properly cited.
}

\section{ABSTRACT}

Background: Radiotherapy is one of the primary modalities of cancer treatment but may associated with short and long-term toxicities. Oral mucositis is frequently encountered in head and neck cancer resulting in unplanned treatment breaks. Few studies emphasized that use of oral glutamine may significantly reduce oral mucositis and other acute toxicities. This study aims to assess the effects of glutamine on oral mucositis in head and neck cancer patients receiving chemo-radiation.

Methods: It is a prospective study carried out in sixty-four head and neck cancer patients attending radiotherapy department for chemo-radiation from January 2018 to May 2019. Patients were randomly assigned into two arms containing 32 subjects in each. All the patients in arm-A were taking oral glutamine supplement of $15 \mathrm{mg}$ once daily, two hours prior to radiation and arm-B serve as the control group. Dose of radiation fixed at 66 Gy in 33 fractions over a period of 7 weeks along with an infusion of weekly cisplatin $\left(40 \mathrm{mg} / \mathrm{m}^{2}\right)$. Patients were evaluated regarding onset, severity and the recovery period of mucositis.

Results: Oral mucositis appeared at around 5th week in arm-A and 3rd week in arm-B ( $\mathrm{p}<0.0001)$. Number of patients with mucositis is significantly less in arm-A (75\%, 24 out of 32) as compared to arm-B $(96.8 \%)(\mathrm{p}=0.0310)$. The time required for healing of mucositis is significantly less than 1 week in arm-A compared to $\sim 2$ weeks in the arm-B (p<0.0001).

Conclusions: Oral glutamine when given prior to radiation results in delayed onset of oral mucositis with decreased severity and an early healing period.

Keywords: Oral glutamine, Head and neck cancer, Mucositis, Radiation toxicities

\section{INTRODUCTION}

Chemo-radiation is one of the multimodality treatments for head and neck cancers. However, the toxicities of treatment are often severe and difficult to manage. ${ }^{1}$ Oncologists frequently encounter oral mucositis, which can be severe and cause hospitalization or unplanned breaks in radiotherapy (RT). ${ }^{2,3}$ Incidence of radiationinduced oral mucositis is $80 \%$ to $91 \%$ in head and neck cancer patients, with or without chemotherapy., ${ }^{4,5}$ This toxicity is due to progressive thinning of the oral mucosa to form erythematous patches which leads to ulceration with severe pain and swallowing disability. This may result in discontinuation of chemo- radiation, use of analgesics or Ryle's tube dependency.

Non-pharmacologic approaches for the prevention of oral mucositis, including glutamine and several other agents, have been investigated. ${ }^{6}$ There is inadequate evidence confirming the advantage of glutamine as the research results have been inconsistent. ${ }^{2,7}$ 
Glutamine is an essential amino acid critical to the regulation of protein synthesis, respiratory fueling and cellular energy and signaling in cancer cells. The skeletal muscle accounts for $90 \%$ of the glutamine synthesized in the body, with the rest released by the lungs and the brain. ${ }^{8}$ In catabolic states of injury or during periods of rapid growth or stress, glutamine becomes conditionally essential, and oral supplementation is necessary. Human cancer cell lines exhibit a 5 to 10 -fold faster rate of glutamine consumption than non-malignant cells. ${ }^{9}$ When the body is under stress and experiencing high metabolic demands, the intracellular glutamine pool is approximately halved. ${ }^{10}$ This can have a negative impact on the function of host tissues that are dependent upon adequate stores of glutamine for optimal functioning. ${ }^{11,12}$

Glutamine may decrease mucous membrane injury induced by radiation by altering the inflammatory response. Glutathione, a byproduct of glutamine metabolism protects against oxidant injury. Glutathione is an antagonist to prostaglandin E2 production, which is a strong inflammatory mediator. A pilot trial in China demonstrated that oral glutamine suspension may significantly reduce the duration and severity of oral mucositis during radiotherapy. ${ }^{13}$ However, an adequately powered randomized clinical study required to establish these findings has not been carried out so far, to the best of our knowledge.

So, in this study we have evaluated the effects of oral glutamine on the onset, severity and the recovery period of radiation induced mucositis in head and neck cancer patients receiving chemo-radiation.

\section{METHODS}

This study was conducted in the Department of Radiotherapy of Maharaja Krishna Chandra Gajapati Medical College, Berhampur, Odisha situated in the eastern part of India. In this department, all the cancer patients were treated in conventional technique by Co60 radiotherapy machine. Our study includes biopsy proven head and neck cancer patients attending radiotherapy department for chemo-radiation. Informed consent was obtained from all the patients satisfying eligibility criteria. After we obtained consent, consecutive patients were randomly assigned one after the other into either treatment arm-A, which was the glutamine arm, or arm$\mathrm{B}$, which was the control arm. Because the study protocol had determined an equal number of patients for each arm of the study, a block randomization protocol was used. To avoid bias, the randomization sequence was obtained in sealed envelopes from a statistician. Patients in arm-A were advised to take glutamine $15 \mathrm{mg}$ (1 sachet) in 100 $\mathrm{ml}$ of water 2 hours before commencement of radiotherapy and also on radiation off days at the same time. Patients in arm-B served as control group.

\section{Study subject}

All biopsy proven (squamous cell carcinoma) cases of head and neck cancer patients who received chemoradiation with a dose of $66 \mathrm{~Gy}$ in 33 fractions, 5 fractions a week with 2 Gy per fraction along with weekly cisplatin in a dose of $40 \mathrm{mg} / \mathrm{m}^{2}$ on Monday every week during the study period from January 2018 to May 2019.

\section{Inclusion criteria}

Age groups- 20 to 80 year, both males and females, histopathologically proven squamous cell carcinoma of the head and neck, primary tumor in stage T2, T3, or T4; regional node of any $\mathrm{N}$ status; without distant metastases, patients with Eastern Cooperative Oncology Group performance and score 0,1 or 2 were included.

\section{Exclusion criteria}

Patients with unscheduled treatment gaps of $>2$ weeks for reasons other than treatment toxicity; patients with history of second primary cancer; presence of any chronic systemic illness; previous history of chemotherapy or radiotherapy were excluded.

\section{Data collection}

Patient's information like socio-demographic and clinical profile, sub-site wise distribution of the disease, onset of mucositis, its grade and recovery period were obtained in a predesigned case format from head and neck cancer patients those provided written consent.

\section{Statistical analysis}

All the data were collected, summarized, and analyzed by SPSS version 16 IBM SPSS Statistics for Windows, (IBM Corp., Armonk, N.Y., USA). The data were presented by their number and percentage. The categorical data were analyzed and compared by Chisquare test. Level of statistical significance was considered as $\mathrm{p}<0.05$.

\section{RESULTS}

A total of 64 patients were included in this study and randomized into two arms with 32 subjects in each group. arm-A (glutamine arm) consisted of 29 (90.6\%) male as compared to $23(71.8 \%)$ in the control group. Mean age is $55.28 \pm 10.65$ and $52.16 \pm 11.73$ year in the case and control arms respectively. According to the tumor sub-site highest number of patients, 23 (71.8\%) in arm-A and 24 (75\%) in arm-B had cancer in the oral cavity. Factors like age $(p=0.3297)$, sex $(p=0.109)$ and sub-site $(p=0.6113)$ of cancer in both the groups were comparable as the $\mathrm{p}$ value for all these three variables were not statistically significant (Table 1). 
Table 1: Comparison of age, gender and sub-sites of head and neck cancer in arm-A (cases: with glutamine) and arm-B (control: without glutamine).

\begin{tabular}{|c|c|c|c|}
\hline Variables & $\begin{array}{l}\text { Arm-A (cases) } \\
(\mathrm{n}=32)\end{array}$ & $\begin{array}{l}\text { Arm-B (control) } \\
(\mathrm{n}=32)\end{array}$ & $P$ value \\
\hline Age (in years) Mean \pm SD & $55.28 \pm 10.65$ & $52.16 \pm 11.73$ & 0.3297 \\
\hline Gender & $\mathbf{N}(\%)$ & $\mathbf{N}(\%)$ & \multirow{3}{*}{$\chi^{2}$ test $=2.560 ; \mathrm{p}=0.109$} \\
\hline Male & $29(90.63)$ & $23(71.88)$ & \\
\hline Female & $3(9.37)$ & $9(28.12)$ & \\
\hline \multicolumn{4}{|c|}{ Sub-sites of head and neck cancer } \\
\hline Oral cavity & $23(71.88)$ & $24(75.0)$ & \multirow{5}{*}{$\chi^{2}$ test $=2.688 ; p=0.6113$} \\
\hline Oropharynx & $3(9.37)$ & $3(9.37)$ & \\
\hline Hypopharynx & $4(12.5)$ & $2(6.26)$ & \\
\hline Larynx & $1(3.13)$ & $33(9.37)$ & \\
\hline Nasopharynx & $1(3.13)$ & 0 & \\
\hline
\end{tabular}

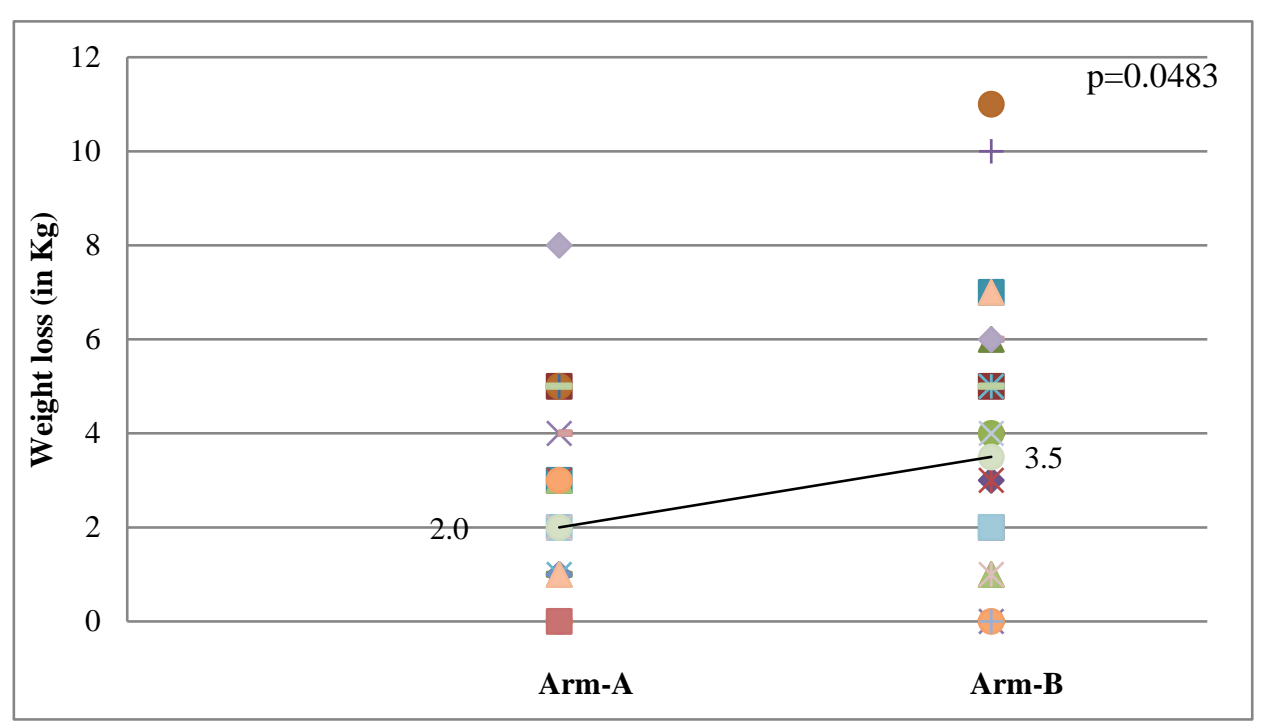

Figure 1: Comparison of weight loss after radiotherapy in head and neck cancer patients in arm-A (cases: with glutamine) and arm-B (control: without glutamine), line joining the median value.

Loss of weight after completion of radiation was due to decreased food intake as a result of mucositis and other acute toxicities. An average weight loss of $2.44 \pm 1.88 \mathrm{~kg}$ was found in arm-A with median at $2 \mathrm{~kg}$ and in arm-B it was $3.66 \pm 2.86 \mathrm{~kg}$ and median value was $3.5 \mathrm{~kg}$ $(p=0.0483)$. The comparison of weight loss after radiotherapy in both the groups of patients has been illustrated in Figure 1.

Mucositis appeared during an average period of 5 weeks (median) from start of treatment in glutamine arm and 3 weeks (median) in control arm. In arm-A mucositis was seen in $24(75 \%)$ patients and in control arm it was 31 $(96.8 \%)$. Further when the duration from appearance of grade 3 and 4 mucositis to its complete healing is compared in both the groups, the recovery period was 1 week and 2 weeks in arm-A and arm-B respectively.

\section{DISCUSSION}

Oral mucositis in head and neck irradiation still remains a challenge and matter of concern. Different interventions are currently practiced with varying benefits, but there is no consensus on the most effective way to prevent or treat this distressing complication. ${ }^{14}$

Although basic oral care maintains mucosal health, little evidence suggests that it can reduce the onset and severity of mucositis as it is radiation induced. ${ }^{15}$ Agents like Nacetyl cysteine, amifostine, and systemic or topical antimicrobial formulations have been formulated for oral mucositis, though without encouraging results. ${ }^{16}$

According to the therapy oncology group double-blind study, subcutaneous use of granulocyte macrophage colony-stimulating factor failed to reduce oral mucositis. ${ }^{17}$ The recombinant form of fibroblast growth factor called keratinocyte growth factor, or palifermin, has been studied in a group of patients with hematologic malignancies who required total-body irradiation with high-dose chemotherapy and blood stem cell support, palifermin reduced the onset and severity of oral mucositis. Therefore, the US Food and Drug 
Administration has approved palifermin for this particular indication alone. ${ }^{18}$

Most recently, Tsujimoto et al reported that glutamine significantly decreased the severity of mucositis in the oral cavity, pharynx, and larynx induced by chemoradiation in patients with head and neck cancers. ${ }^{19}$ However, they did not include any patients with oral cavity cancer and the dosage of glutamine was $30 \mathrm{~g} / \mathrm{d}$ divided into 3 doses. A previous study that demonstrated positive effects in alleviation of oral mucositis used a dose range of 10 to $30 \mathrm{~g} / \mathrm{d} .^{20}$ We supplemented our patients with $15 \mathrm{gm}$ once daily, 2 hours prior to radiation and oral cavity cancers were also included along with other sub-sites in our study.

In the study of Huang et al, they included patients with various head and neck cancers, although the majority had oral cavity cancer $(65.6 \%, \mathrm{n}=42)$. This may contribute to a higher incidence or severity of oral mucositis because the oral mucosa is in a high-dose area for these patients. Under such circumstances, they tested whether oral glutamine might help relieve the incidence and severity of oral mucositis with recorded toxicity up to $70 \mathrm{~Gy} .{ }^{21}$ In our study also oral cavity is the most common sub-site $(73.4 \%, \mathrm{n}=47)$ and the radiation dose is limited upto 66 Gy.

In a study carried out at A. H. Regional cancer center, they evaluated 162 patients with locally advanced head and neck cancer treated with concomitant chemoradiation. From their analysis, $1.2 \%$ of patients in the glutamine arm versus $55.5 \%$ of patients in the control arm developed mucositis at the end of the third week. At the sixth week, $34.5 \%$ of patients in the glutamine arm developed grade- 2 mucositis, and none of the patients developed G3 mucositis. However, in the control arm, $54.32 \%$ of patients had G2 mucositis as early as the fourth week, and $92.35 \%$ of patients had grade- 3 mucositis by the sixth week. ${ }^{21}$ Our study $75 \%$ and $96 \%$ of patients developed mucositis in arm-A and arm-B respectively.

Huang et al postulated that a reciprocal causation occurs between the decreased BMI and the severity of oral mucositis. When more severe mucositis causes an inability to eat, inadequate oral intake decreases the body weight and hence the BMI. Debilitated nutritional status from weight loss reduces the healing capability, and therefore leads to more severe mucositis. Under these circumstances, an oral nutritional supplement such as glutamine alone may not reverse the condition. The internal mechanism of this reciprocal causation is not clear and further laboratory exploration is warranted. ${ }^{22}$

In another study it is showed that oral glutamine delays the development of mucositis. The mean time of onset of mucositis is significantly delayed in patients who received glutamine with $\mathrm{p}<0.001$. The mean duration of grade 3 mucositis or worse (grade 3 and grade 4) was significantly less (6.6 days vs. 9.2 days) in the glutamine arm. ${ }^{23}$ Our study revealed similar result of recovery period of oral mucositis to be 1 week and 2 weeks in the glutamine arm and control arm respectively.

Huang et al in their randomized control trial concluded that, despite several positive results from the literature, oral glutamine failed to reduce RT-induced oral mucositis or neck dermatitis in patients with head and neck cancer. However, the decrease in BMI strongly correlated with a higher severity of oral mucositis during RT. ${ }^{22}$ In contrast our study showed a significant improvement in oral mucositis and also less weight loss in the glutamine arm.

\section{CONCLUSION}

Oral mucositis is really a matter of concern in head and neck cancer patients receiving chemo-radiation. Most of the patients develop mucositis but glutamine has a significant effect on delaying the onset, decreasing the severity and early recovery of oral mucositis in head and neck cancer patients who were undergone chemoradiation.

\section{ACKNOWLEDGEMENTS}

The statistical data analysis and manuscript preparation has been carried out at Multi-Disciplinary Research Unit (MRU), M.K.C.G. Medical College, Berhampur, Odisha.

\section{Funding: No funding sources \\ Conflict of interest: None declared}

Ethical approval: The study was approved by the Institutional Ethics Committee of M.K.C.G. Medical College, Berhampur, Odisha

\section{REFERENCES}

1. Dose AM. The symptom experience of mucositis, stomatitis, and xerostomia. Semin Oncol Nurs. 1995; 11:248-55.

2. Saadeh CE. Chemotherapy- and radiotherapyinduced oral mucositis: review of preventive strategies and treatment. Pharmacotherapy. 2005;25(4):540-4.

3. Vera-Llonch M, Oster G, Hagiwara M, Sonis S. Oral mucositis in patients undergoing radiation treatment for head and neck carcinoma. Cancer. 2006;106(2):329-36.

4. Elting LS, Cooksley CD, Chambers MS, Garden AS. Risk, outcomes, and costs of radiation-induced oral mucositis among patients with headand- neck malignancies. Int $\mathbf{J}$ Radiat Oncol Biol Phys. 2007;68(4):1110-20.

5. Trotti A, Bellm LA, Epstein JB, Frame D, Fuchs HJ, Gwede CK, et al. Mucositis incidence, severity and associated outcomes in patients with head and neck cancer receiving radiotherapy with or without chemotherapy: a systematic literature review. Radiother Oncol. 2003;66(3):253-62. 
6. Worthington HV, Clarkson JE, Eden OB. Interventions for preventing oral mucositis for patients with cancer receiving treatment. Cochrane Database Syst Rev. 2007(4):Cd000978.

7. Stokman MA Vries EG. Preventive intervention possibilities in radiotherapy and chemotherapyinduced oral mucositis: results of meta-analyses. J Dent Res. 2006;85(8):690.

8. Newsholme P, Lima MMR, Procopio J, Pithon-Curi TC, Doi SQ, Bazotte RB, et al. Glutamine and glutamate as vital metabolites. Braz J Med Biol Res. 2003;36:153-63.

9. Souba WW. Glutamine and cancer. Ann Surg. 1993;218(6):715-28.

10. Furst P, Albers S, Stehle P. Evidence for a nutritional need for glutamine in catabolic patients. Kidney Int. 1989;27:287-92.

11. Ardawi MS, Newsholme EA. Glutamine metabolism in lymphocytes of the rat. Biochem $\mathrm{J}$. 1983;212:835-42.

12. Klimberg VS, Souba WW, Dolson DJ, Salloum RM, Hautamaki RD, Plumley DA, et al. Prophylactic glutamine protects the intestinal mucosa from radiation injury. Cancer. 1990;66:62-8.

13. Silverman S Jr. Diagnosis and management of oral mucositis. J Support Oncol. 2007;5(suppl 1):13-21.

14. Mead GM. Management of oral mucositis associated with cancer chemotherapy. Lancet. 2002;359:815-6.

15. Sonis ST, Elting LS, Keefe D, Peterson DE, Schubert M, Hauer-Jensen $M$, et al. Perspectives on cancer therapy-induced mucosal injury: pathogenesis, measurement, epidemiology, and consequences for patients. Cancer. 2004;100:1995-2025.

16. Symonds RP, McIlroy P, Khorrami J, Paul J, Pyper $\mathrm{E}$, Alcock SR, et al. The reduction of radiation mucositis by selective decontamination antibiotic pastilles: A placebo-controlled double-blind trial. $\mathrm{Br}$ J Cancer. 1996;74:312-7.

17. Rovirosa A, Ferre J, Biete A. Granulocyte macrophage-colony-stimulating factor mouthwashes heal oral ulcers during head and neck radiotherapy. Int J Radiat Oncol Biol Phys. 1998;41:747-54.

18. Antunes HS, de Azevedo AM, da Silva Bouzas LF, Adão CA, Pinheiro CT, Mayhe R, et al. Low-power laser in the prevention of induced oral mucositis in bone marrow transplantation patients: A randomized trial. Blood. 2007;109:2250-5.

19. Tsujimoto T, Yamamoto Y, Wasa M, Takenaka Y, Nakahara S, Takagi T, et al. L-Glutamine decreases the severity of mucositis induced by chemoradiotherapy in patients with locally advanced head and neck cancer: a double-blind, randomized, placebo-controlled trial. Oncol Rep. 2015;33(1):33-9.

20. Leung HW, Chan AL. Glutamine in alleviation of radiation-induced severe oral mucositis: a metaanalysis. Nutr Cancer. 2016;68(5):734-42.

21. Pattanayak L, Panda N, Dash MK, Mohanty S, Samantaray S. Management of ChemoradiationInduced Mucositis in Head and Neck Cancers with Oral Glutamine. J Global Oncol Am Society Clin Oncol. 2016;2;4200-6.

22. Huang CJ, Huang MY, Fang PT, Chen F, Wang YT, Chen $\mathrm{CH}$, et al., Randomized double-blind, placebocontrolled trial evaluating oral glutamine on radiation-induced oral mucositis and dermatitis in head and neck cancer patients. Am J Clin Nutr. 2019;109:606-14.

23. Chattopadhyay S, Saha A, Azam M, Mukherjee A, Sur PK. Role of oral glutamine in alleviation and prevention of radiationinduced oral mucositis: A prospective randomized study. South Asian J Cancer. 2014;3:8-12.

Cite this article as: Das BP, Patra J. Effect of glutamine supplement on chemo-radiation induced mucositis in head and neck cancer patients: a prospective study. Int J Basic Clin Pharmacol 2020;9:417-21. 